W.P. Holland

\title{
The nuclei of atoms in Periods 4, 5, 6 and 7
}

Abstract. In my previous paper on " The nucleus of an atom and the periodicity of the elements" I showed that the nucleus of an atom represented by neutron-proton units in the form of truncated tetrahedra could be assembled to show the periodicity of elements as suggested by Mendeleev. ${ }^{1}$ The concept was simple for Periods 1, 2 and 3 (namely all the elements from hydrogen to argon). Periods 4 and 5 had the added complexity of the ten transition elements and Period 6 had even more complexity with not only ten transition elements but also fourteen rare-earth elements. The following notes on Periods 4, 5 and 6 show that the nuclei of these elements can be extensions of the octahedral structure of the nuclei of atoms. Period 7, of which we have only a few examples on Earth, actinium to americium, can follow the same building up of 48 neutron-proton units over the large radon nucleus to the next inert gas element with atomic number 134 .

Nanotechnology Perceptions 13 (2017) 123-127

doi: 10.4024/N14HO17N.ntp.13.02 